\title{
Diagnostic value of T-SPOT.TB interferon- $\gamma$ release assays for active tuberculosis
}

\author{
LIPING YAN*, HEPING XIAO*, MIN HAN and QING ZHANG \\ Department of Tuberculosis, Shanghai Pulmonary Hospital, Tongji University \\ School of Medicine, Shanghai 200433, P.R. China
}

Received August 4, 2014; Accepted April 13, 2015

DOI: $10.3892 / \mathrm{etm} .2015 .2463$

\begin{abstract}
The aim of the present study was to evaluate the diagnostic value of interferon- $\gamma$ release assays for the detection of active tuberculosis (ATB) in patients previously vaccinated with Bacillus Calmette-Guérin (BCG). In total, 540 patients underwent the T-SPOT.TB test, including 295 patients with active pulmonary TB (PTB), 52 patients with active extrapulmonary TB (EPTB), 11 individuals with inactive TB and 182 non-TB cases. Simultaneously, 186 patients with ATB, including PTB and EPTB cases, and 125 non-TB patients underwent tuberculin skin tests (TST). Associations between the sensitivity of the T-SPOT.TB assays and lung lesion severity, positive smear grade, disease site and the duration of anti-TB treatment were evaluated. The sensitivity and specificity values of the T-SPOT.TB assay for diagnosing ATB were 76.66 and $76.37 \%$, respectively, and the positive rate in the inactive TB test results was significantly lower $(23.63 \%$; $\mathrm{P}<0.001)$. The diagnostic sensitivity was higher in the PTB cases when compared with the EPTB cases $(\mathrm{P}=0.01)$. Furthermore, the diagnostic sensitivity of the ATB cases undergoing anti-TB treatment was significantly lower when compared with the cases not undergoing treatment $(\mathrm{P}=0.002)$, and the sensitivity gradually decreased with the treatment duration $(\mathrm{P}=0.01)$. In addition, a statistically significant difference was identified in the specificity between the T-SPOT.TB assay and the TST (76.37 vs. $51.15 \%$; $\mathrm{P}<0.001$ ), whereas the sensitivity values did not differ significantly (76.66 vs. $75.56 \%$ ). Therefore, the results indicated that the T-SPOT.TB assay is a promising diagnostic test for active PTB in a BCG-vaccinated population, and should replace the TST. As the administration of anti-TB
\end{abstract}

Correspondence to: Professor Qing Zhang, Department of Tuberculosis, Shanghai Pulmonary Hospital, Tongji University School of Medicine, 507 Zhengmin Road, Shanghai 200433, P.R. China

E-mail: zhangingbm123@163.com

*Contributed equally

Key words: interferon- $\gamma$ release assays, T-SPOT.TB, tuberculin skin test, pulmonary tuberculosis, extrapulmonary tuberculosis treatment resulted in a lower sensitivity to the diagnostic test, the T-SPOT.TB assay may also be suitable for the assessment of treatment outcomes.

\section{Introduction}

Tuberculosis (TB) has the greatest mortality rate among all infectious diseases, which is primarily due to the current lack of effective diagnostic methods (1). The gold standard for the diagnosis of pulmonary TB (PTB) is the identification of Mycobacterium tuberculosis via in vitro culture. However, the cultures are prone to detection limits and can be time consuming, in addition to the difficulty of obtaining clinical isolates, particularly in cases of extrapulmonary TB (EPTB) (2); thus, clinicians often have to rely on immunoassays for diagnosis. In addition, the diagnostic value of the tuberculin skin test (TST) is limited due to the cross-reactivity of the purified protein derivative from the Bacillus Calmette-Guérin (BCG) vaccination and non-tuberculous Mycobacteria (NTM) species (3).

Previously, interferon $-\gamma$ release assays (IGRAs), which are based on the level of IFN- $\gamma$ secreted by T cells in response to $M$. tuberculosis specific antigens, have demonstrated a superior diagnostic performance over the conventional TST (4-6). These specific antigens, which include early secreted antigenic target (ESAT-6; $6 \mathrm{kDa}$ ) and culture filtrate protein (CFP-10; $10 \mathrm{kDa}$ ), are coded by region of difference 1 , which is present in the genome of M.tuberculosis and absent in BCG or NTM strains, with the few exceptions of M. marinum, M. kansasii, M. gordonae and M. szulgai $(7,8)$. There are two IGRA tests currently available on the market, namely Quanti FERON-Tb Gold (Cellestis, Carnegie, Australia) and T-SPOT.TB (Oxford Immunotec, Ltd., Oxford, UK). The Quanti FERON-Tb Gold assay measures the level of IFN- $\gamma$ using an enzyme-linked immunosorbent assay method, while the T-SPOT.TB assay identifies $\mathrm{T}$ cells secreting IFN- $\gamma$ using an enzyme-linked immuno-spot (ELISpot) assay technique, which combines the immune enzymatic methods with a short-term culture of $\mathrm{T}$ cells. One of the advantages of the ELISpot technique is the high sensitivity, which enables the detection of a single M. tuberculosis antigen-activated T cell. Although a number of studies have evaluated the performance of IGRAs for the diagnosis of latent TB infections (9-11), studies assessing the value of IGRAs for the diagnosis of active TB (ATB) are 
limited, particularly those including a large patient sample population. Certain studies have indicated that quantitative T-cell responses, as measured with the T-SPOT.TB assay, may indicate a mycobacterial burden and disease activity, however, are unable to discriminate between active and latent TB (12). By contrast, other studies have observed that the T-SPOT.TB assay has a high sensitivity for diagnosing ATB (13). To date, the diagnostic value of the T-SPOT.TB assay for ATB remains controversial, and since China is a country with a serious TB burden, a prospective study was performed to evaluate the diagnostic reliability of the T-SPOT.TB assay in 540 patients with and without ATB, as well as inactive TB participants.

The aim of the present study was to investigate the factors affecting the performance of T-SPOT.TB assays, and to elucidate whether T-SPOT.TB tests are sufficiently sensitive and specific for the diagnosis of ATB, without the requirement of a positive $M$. tuberculosis culture or the presence of caseating granuloma in biopsy specimens, thereby providing the basis for optimized treatment regimens and the avoidance of further transmissions.

\section{Patients and methods}

Patient inclusion and diagnosis. In the prospective study, 540 consecutive patients, who were admitted to the Shanghai Pulmonary Hospital (Shanghai, China) between December 2011 and September 2012, were enrolled due to a suspected case of TB. All the subjects were HIV-negative and had received a BCG vaccination in early childhood or during adolescence. A standard questionnaire was completed for each patient, which included basic demographic data, a history of TB contacts, previous TB history, current TB symptoms, administration of anti-TB treatment, as well as underlying diseases and concurrent immunosuppressive therapies. All 540 patients underwent a T-SPOT.TB test at enrollment, in addition to routine clinical, microbiological, pathological and radiographic examinations, including physical examination, sputum smear and culture, and chest computed tomography. Final classifications were obtained through analysis of the patients' medical records, in addition to laboratory and chest X-ray results. Definite cases of TB were confirmed based on positive cultures of M.tuberculosis from the sputum or the presence of caseating granuloma in the biopsy specimens. Clinical TB was diagnosed if there were TB-specific radiographic observations, including nodules, patches, cavity and tree-in-bud sign, and appropriate responses to anti-TB treatments. Patients classified with ATB included those with confirmed TB or clinical TB, and were further classified into cases of PTB and EPTB. Inactive TB was diagnosed if the patient had a history of TB, however, the disease was not active at the time of admission. Patients were classified as non-TB if there was a confirmed alternative diagnosis without a history of TB. The study was approved by the Ethical Committee of Shanghai Pulmonary Hospital, and informed consent was obtained from all the participants.

$X$-ray criteria for the lesion severity scores. Chest X-rays of the patients were divided into six lung fields (Fig. 1) and the severity of the lung lesions was scored based on the following
Table I. Criteria for lesion severity.

\begin{tabular}{lc}
\hline Criteria & Severity score \\
\hline Disease & \\
No disease & 0 \\
$<50 \%$ of area affected & 1 \\
$\geq 50 \%$ of area affected & 2 \\
Cavitation & \\
No cavitation & 0 \\
Single cavity & \\
$<2$ cm diameter & 0.25 \\
$2-4$ cm diameter & 0.5 \\
$>4$ cm diameter & 1.0 \\
Multiple cavities & \\
Largest $<2$ cm diameter & 0.5 \\
Largest $2-4$ cm diameter & 1.0 \\
Largest $>4$ cm diameter & 2.0 \\
\hline
\end{tabular}

factors: i) Range of the lung field foci; and ii) number/size of the cavities (Table I). The final lesion severity score was the sum of the scores from the six lung fields (every lung field $=\mathrm{i}+\mathrm{ii}$, as outlined in Table I). Scores of $\leq 2.5$ were classified as mild, while those ranging between 2.5 and 6 were defined as moderate, and $\geq 6$ points was classified as severe.

Sputum smear grade. The sputum smears were stained by Ziehl-Neelsen staining (Merck Millipore, Darmstadt, Germany), and were observed under a fluorescent microscope (EPI LBX-2002H; Shanghai Longway Optical Instruments Co., Ltd., Shanghai, China). Sputum smear samples were graded as follows: Smear $3+, \geq 10$ acid-fast bacilli (AFB) per 1 high-power field (HPF); smear 2+, 1-9 AFB per $1 \mathrm{HPF}$; and smear 1+, 10-99 AFB per $100 \mathrm{HPF}$.

T-SPOT.TB assay. T-SPOT.TB tests were performed in accordance with the manufacturer's instructions outlined in the assay kit (Oxford Immunotec Ltd.). Blood samples were collected immediately prior to the tests in order to avoid potential interferences, and patients who received blood transfusions or underwent positron emission tomography-computed tomography scans within 1 week of the test were recommended to undergo a second test two weeks later. Peripheral blood mononuclear cells (PBMCs) were separated from blood samples using Ficoll-Hypaque gradient centrifugation at $400 \mathrm{x} \mathrm{g}$ for $30 \mathrm{~min}$ at $20^{\circ} \mathrm{C}$. Briefly, PBMCs were seeded on precoated IFN- $\gamma$ ELISpot plates and incubated with media without an antigen (as a negative control), media containing peptide antigens derived from ESAT-6 (labeled panel A) or peptide antigens derived from CFP-10 (labeled panel B), or media containing phytohemagglutinin (as a positive control) in a $5 \% \mathrm{CO}_{2}$ atmosphere at $37^{\circ} \mathrm{C}$ for $20 \mathrm{~h}$. After counting the number of spot-forming cells, the results of the T-SPOT.TB assay were considered to be positive if panels A or B, or both panels, exhibited six or more spots as compared with the negative control, or if the number of spots was at least two times greater than that of the negative control. 
A

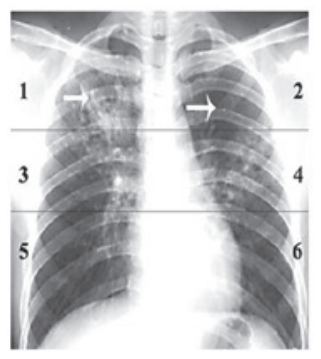

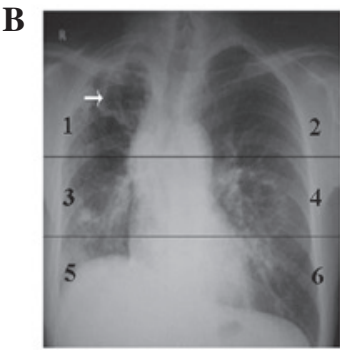
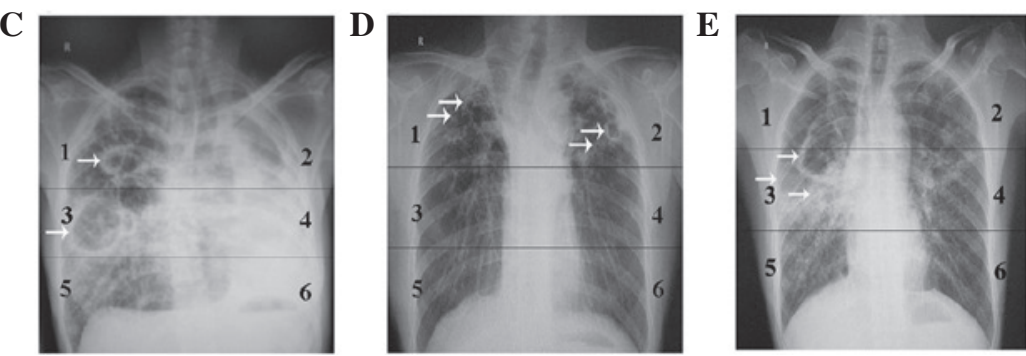

Figure 1. X-ray images showing the lesion severity scoring. The white arrows indicate the lesions and cavities. (A) Field $1, \geq 50 \%$ of area affected $=$ score 2 ; Field $2,<50 \%$ of area affected $=$ score 1 . (B) Field 1 , single cavity, $<2 \mathrm{~cm}$ diameter $=$ score 0.25 . (C) Field 1 , single cavity, $2-4 \mathrm{~cm}$ diameter $=$ score 0.5 ; Field 3 , single cavity, $>4 \mathrm{~cm}$ diameter $=$ score 1 . (D) Field 1 , multiple cavities, the largest $<2 \mathrm{~cm}$ diameter $=$ score 0.5 ; Field 2 , multiple cavities, the largest $2-4 \mathrm{~cm}$ diameter $=$ score 1 . (E) Field 3, multiple cavities, the largest $>4 \mathrm{~cm}$ diameter $=$ score 2.

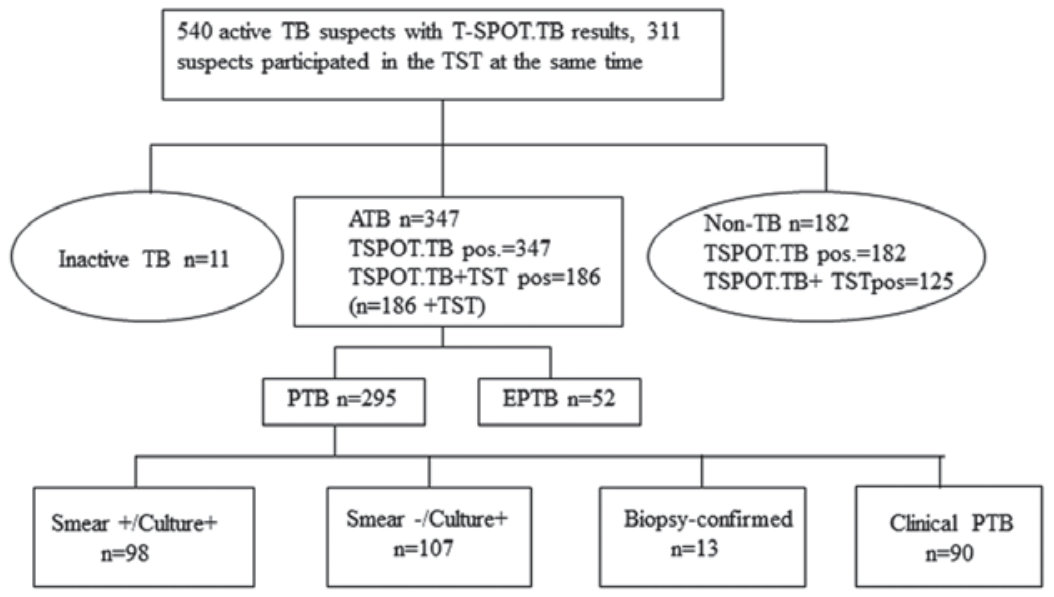

Figure 2. Flowchart of the study population. A total of 540 subjects who were suspected of having ATB were recruited for the T-SPOT.TB test, and 311 individuals underwent a TST simultaneously. TB, tuberculosis; ATB, active tuberculosis; TST, tuberculin skin test; PTB, pulmonary tuberculosis; EPTB, extrapulmonary tuberculosis.

TST. Medical staff of Shanghai Pulmonary Hospital conducted the TSTs. In total, five tuberculin units of purified protein derivative were injected intradermally into the volar aspects of the left forearm, according to the Mantoux method, and the skin induration was examined after $72 \mathrm{~h}$. If the induration diameter was $<5 \mathrm{~mm}$, the TST was considered to be negative, while if the diameter was $\geq 5 \mathrm{~mm}$, or in the case of blister development, the TST was considered to be positive.

Statistical analysis. Statistical analyses were conducted using SPSS statistical software (SPSS Statistics 17.0; SPSS, Inc., Chicago, IL, USA). Assuming a positive rate of $75 \%$ for the T-SPOT.TB tests in the patients with ATB, a sample size of 133 patients with TB was required to conduct a power test. Sensitivity, specificity, positive predictive value (PPV), negative predictive value (NPV), positive likelihood ratio (LR+) and negative likelihood ratio (LR-) values, together with their 95\% confidence intervals (CIs), were calculated to evaluate the diagnostic performance of the T-SPOT.TB test, according to the binomial distribution. $\mathrm{P}<0.05$ was considered to indicate a statistically significant difference. Comparisons of positive rates between the different defined groups were analyzed using Pearson's $\chi^{2}$ test or Fisher's exact test. The difference between the paired proportions was analyzed with McNemar's test, while the Wilcoxon rank test was used for the comparison of continuous variables. Risk factors for individual assay positivity were evaluated using odds ratios in a univariate analysis. In addition, using a multiple regression analysis model, the TSPOT.TB test was selected as a dependent variable, while over the median age ( $>50$ years), gender, clinical symptoms, anti-TB treatment administration and disease site (PTB or EPTB) were selected as independent variables. The concordance of agreement between the TSPOT.TB test and TST results was assessed using Cohen's $\kappa$ coefficient, where $\kappa>0.75$ inferred an excellent agreement, $0.4<\kappa<0.75$ indicated moderate agreement and $\kappa<0.4$ inferred a poor agreement.

\section{Results}

Demographic profiles of the patients. A total of 540 consecutive patients, who required a TB differential diagnosis, were initially enrolled in the study. The sample population included 295 active PTB cases (including 89 patients undergoing anti-TB treatment), 52 active EPTB cases (including 6 cases of osteoarticular TB, 13 cases of tuberculous lymphadenitis and 33 cases of tuberculous pleurisy), as well as 11 inactive TB cases and 182 patients without TB (non-TB) (Fig. 2). Patients with EPTB in combination with PTB were included in the PTB group. The baseline characteristics of the enrolled patients are summarized in Table II. The mean ages of the patients with ATB, an inactive form of the disease and the non-TB subjects were $50 \pm 17,56 \pm 16$ and $50 \pm 17$ years, respec- 
Table II. Baseline characteristics of the enrolled patients.

\begin{tabular}{lcccc}
\hline & \multicolumn{2}{c}{ ATB $(\mathrm{n}=347)$} & & \\
\cline { 2 - 4 } Characteristics & PTB $(\mathrm{n}=295)$ & EPTB $(\mathrm{n}=52)$ & Non-TB $(\mathrm{n}=182)$ & Inactive TB $(\mathrm{n}=11)$ \\
\hline Age $^{\mathrm{a}}$, years & $50 \pm 17(5-87)$ & $51 \pm 16(9-78)$ & $56 \pm 16(15-88)$ & $50 \pm 17(19-75)$ \\
Gender (male:female), $\mathrm{n}$ & $216: 79$ & $32: 20$ & $122: 60$ & $5: 6$ \\
Diabetes mellitus, $\mathrm{n}$ & 30 & 0 & 10 & 0 \\
Malignant disease, $\mathrm{n}$ & 1 & 1 & 1 & 0 \\
Autoimmune diseases, $\mathrm{n}$ & 4 & 0 & 1 & 0 \\
Liver disease, $\mathrm{n}$ & 1 & 0 & 0 & 0 \\
Chronic renal failure, $\mathrm{n}$ & 1 & 0 & 0 & 0
\end{tabular}

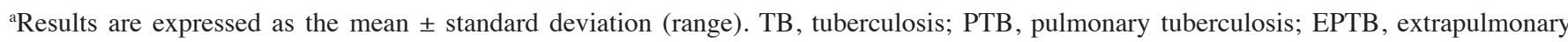
tuberculosis; ATB, active tuberculosis.

Table III. T-SPOT.TB results in the non-TB subgroups.

\begin{tabular}{|c|c|c|c|c|c|}
\hline Condition & Age, years & $\begin{array}{c}\text { Gender } \\
\text { (male:female), } \mathrm{n}\end{array}$ & $\begin{array}{c}\text { T-SPOT.TB }(+), \\
\mathrm{n}(\%)\end{array}$ & $\begin{array}{c}\text { T-SPOT.TB (-), } \\
\mathrm{n}(\%)\end{array}$ & Total, $\mathrm{n}$ \\
\hline Pulmonary NTM disease & $51 \pm 15(32-78)$ & $5: 4$ & $4(44.44)$ & $5(55.56)$ & 9 \\
\hline Lung cancer & $48 \pm 16(35-80)$ & $35: 26$ & $16(26.23)$ & $45(73.33)$ & 61 \\
\hline Pneumonia & $49 \pm 17(21-78)$ & $47: 40$ & $16(18.39)$ & $71(81.61)$ & 87 \\
\hline Pulmonary mycosis & $51 \pm 17(35-80)$ & $4: 3$ & $2(28.57)$ & $5(71.43)$ & 7 \\
\hline Silicosis & $48 \pm 8(40-57)$ & $7: 0$ & $1(14.29)$ & $6(85.71)$ & 7 \\
\hline Bronchogenic cyst & $49 \pm 4(45-51)$ & $2: 1$ & $1(33.33)$ & $2(66.67)$ & 3 \\
\hline Interstitial lung disease & $50 \pm 2(48-52)$ & $1: 1$ & $0(0)$ & $2(100)$ & 2 \\
\hline Pulmonary embolism & $50 \pm 1(49-51)$ & $1: 1$ & $1(50.00)$ & $1(50.00)$ & 2 \\
\hline Granulomatous vasculitis & 43 & $0: 1$ & $0(0)$ & $1(100)$ & 1 \\
\hline Right lower lobe sequestration & 46 & $1: 0$ & $0(0)$ & $1(100)$ & 1 \\
\hline Lung tissue $\mathrm{X}$ disease & 51 & $0: 1$ & $1(100)$ & $0(0)$ & 1 \\
\hline Myelodysplastic syndrome & 45 & $1: 0$ & $1(100)$ & $0(0)$ & 1 \\
\hline Total & $51 \pm 16(21-81)$ & $104: 78$ & $43(23.63)$ & $139(76.37)$ & 182 \\
\hline
\end{tabular}

Results are expressed as the mean \pm standard deviation (range). TB, tuberculosis; NTM, non-tuberculous Mycobacterium.

tively. Male patients were significantly dominant in the ATB and non-TB groups. Almost $10 \%$ of the PTB patients presented with diabetes mellitus, while in the non-TB patient group, only $5.5 \%$ of the patients had diabetes mellitus. In the EPTB and inactive TB groups, there were no patients with diabetes mellitus, and few additional complications were observed in any of the TB groups. The positive test rate in the non-TB group was $23.63 \%$ (43/182; Table III). Patients with pulmonary NTM disease were determined to have an equal positive and negative T-SPOT.TB ratio, while other diseases, including lung cancer, pneumonia, pulmonary mycosis and silicosis, in the non-TB group exhibited negative T-SPOT.TB test ratios of $71.43-85.71 \%$ (Table III). With regard to the TB patients with ATB and inactive TB (Table IV), $76.66 \%$ of the ATB patients were shown to test positive in the T-SPOT.TB test, which included $77.97 \%$ PTB and 69.23\% EPTB patients. By contrast, in the inactive TB group, only $36.36 \%$ (4/11) of the patients exhibited positive results. These results indicated that the majority of ATB patients produced positive T-SPOT.TB test results. In addition, positive T-SPOT.TB test results in the ATB subgroup were found to be associated with positive results from the bacterial cultures and smear or biopsy-confirmed diagnoses $(\mathrm{P}=0.023)$; however, the test sensitivity was higher in the smear+/culture+/clinical test cases, as compared with the biopsy-confirmed cases. Subsequently, the performance of the T-SPOT.TB assay stratified with the disease site was investigated, and the sensitivity was lower in the EPTB patients when compared with the PTB cases. Notably, in contrast to osteoarticular TB, a higher sensitivity was observed in the patients with tuberculous pleurisy and tuberculous lymphadenitis. The difference among the three groups was statistically significant $\left(\chi^{2}=9.1974, \mathrm{P}=0.01\right.$; Table IV $)$. Moreover, the performance of the T-SPOT.TB assay when stratified with the duration of anti-TB treatment revealed that the test sensitivity in the ATB cases during treatment $(65.17 \%, 58 / 89)$ was significantly lower compared with those not undergoing treatment 
Table IV. T-SPOT.TB results in the TB subgroups.

\begin{tabular}{|c|c|c|c|c|c|}
\hline \multirow[b]{2}{*}{ Parameter } & \multicolumn{2}{|c|}{ T-SPOT.TB, n (\%) } & \multirow[b]{2}{*}{ Total, n } & \multirow[b]{2}{*}{$\chi^{2}$} & \multirow[b]{2}{*}{ P-value } \\
\hline & Positive & Negative & & & \\
\hline ATB group & $264(76.08)$ & $83(23.92)$ & 347 & & \\
\hline Gender & & & & 0.9252 & 0.336 \\
\hline Male & $186(74.70)$ & $63(25.30)$ & 249 & & \\
\hline Female & 78 (79.59) & $20(20.41)$ & 98 & & \\
\hline Age & & & & 3.2241 & 0.072 \\
\hline Older patients (>50 years) & $89(70.63)$ & $37(29.37)$ & 126 & & \\
\hline Younger patients ( $<50$ years) & $175(79.20)$ & $46(20.80)$ & 221 & & \\
\hline Diagnosis & & & & 11.1024 & 0.011 \\
\hline Smear positive TB & $84(85.71)$ & $14(14.29)$ & 98 & & \\
\hline Culture positive TB & $73(73.66)$ & $34(26.34)$ & 107 & & \\
\hline Biopsy-confirmed TB & $9(69.23)$ & $4(30.77)$ & 13 & & \\
\hline Clinical TB & $64(83.12)$ & $13(16.88)$ & 77 & & \\
\hline Site & & & & 1.8852 & 0.170 \\
\hline РТВ & $230(77.97)$ & $65(22.03)$ & 295 & & \\
\hline ЕРТВ & $36(69.23)$ & $16(30.77)$ & 52 & 9.1974 & 0.01 \\
\hline Osteoarticular & $1(16.67)$ & $5(83.33)$ & 6 & & \\
\hline Tuberculous lymphadenitis & $9(69.23)$ & $4(30.77)$ & 13 & & \\
\hline Tuberculous pleurisy & $26(78.79)$ & $7(21.21)$ & 33 & & \\
\hline Anti-TB treatment & & & & 8.5883 & 0.001 \\
\hline Without treatment & $208(80.62)$ & $50(19.38)$ & 258 & & \\
\hline During ATB & $54(60.67)$ & $35(39.33)$ & 89 & 8.5883 & 0.01 \\
\hline Treatment $\leq 1$ month & $17(85.00)$ & $3(15.00)$ & & & \\
\hline Treatment $\leq 1$ year & $29(59.18)$ & $20(40.82)$ & & & \\
\hline Treatment $>1$ year & $8(40.00)$ & $12(60.00)$ & 20 & & \\
\hline Inactive TB & $4(36.36)$ & $7(63.64)$ & 11 & 9.3374 & 0.002 \\
\hline
\end{tabular}

TB, tuberculosis; ATB, active tuberculosis; PTB, pulmonary tuberculosis; EPTB, extrapulmonary tuberculosis.

(80.62\%, 208/258; $\left.\chi^{2}=8.8292, P=0.003\right)$, and the sensitivity gradually decreased with an increasing treatment duration. The difference among the treatment times of $<1$ month, $<1$ year and $>1$ year was statistically significant $\left(\chi^{2}=8.5883\right.$, $\mathrm{P}=0.01$; Table IV).

Factors associated with positive and negative test results. Factors associated with positive and negative test results in the ATB group were evaluated using a multivariate analysis. A median age of $>50$ years, administration of anti-TB treatment and the male gender were demonstrated to be three independent factors associated with negative test results, whereas a diagnosis of PTB was associated with positive test results (Table V).

Consistency between T-SPOT.TB assay and TST. In 311 patients of the total 540 subjects (57.59\%), TST results were also recorded. These cases included 186 ATB patients and 125 non-TB patients (Fig. 1). A statistically significant difference in specificity was identified between the T-SPOT.TB and TST assays (76.37 vs. $51.15 \%, \chi^{2}=21.5487, \mathrm{P}<0.001$ ); however, no statistically significant difference in sensitivity
Table V. Multivariate analysis of factors for T-SPOT.TB results in the ATB subgroups.

\begin{tabular}{lccr}
\hline Factors & OR & $95 \%$ CI & P-value \\
\hline Gender & 0.742 & $0.574-0.959$ & 0.023 \\
Age & 0.590 & $0.393-0.885$ & 0.011 \\
Site & 1.457 & $1.057-2.008$ & 0.022 \\
Anti-TB treatment & 0.610 & $0.474-0.785$ & $<0.001$ \\
\hline
\end{tabular}

TB, tuberculosis; ATB, active tuberculosis; OR, odds ratio; CI, confidence interval.

was observed ( 76.66 vs. $75.56 \%, \chi^{2}=0.0000, \mathrm{P}=0.9987$ ). A poor level of agreement $(\mathrm{n}=311 ; 62.88 \%)$ was observed between the T-SPOT.TB assay and the TST $(\kappa=0.26 ; 95 \% \mathrm{CI}$, $0.18-0.34$ ), and the majority of the discordant results were due to TST-positive and T-SPOT.TB-negative results $(68.31 \%$, $125 / 183$ ). Through stratifying the TST-positive patients into four groups by the step-increased sizes of TST indurations, the 
Table VI. T-SPOT.TB results in the subgroups with different TST skin induration sizes.

\begin{tabular}{|c|c|c|c|}
\hline \multirow[b]{2}{*}{ TST induration size } & \multicolumn{2}{|c|}{ T-SPOT.TB, n (\%) } & \multirow[b]{2}{*}{ Matching results (\%) } \\
\hline & Positive & Negative & \\
\hline \multicolumn{4}{|l|}{ Total, mm } \\
\hline$<5$ & $37(33.33)$ & $74(66.67)$ & 66.67 \\
\hline $5-9$ & $18(48.65)$ & $19(51.35)$ & 48.65 \\
\hline $10-19$ & $80(69.57)$ & $35(30.43)$ & 69.57 \\
\hline$\geq 20$ & $31(64.58)$ & $17(35.42)$ & 64.58 \\
\hline \multicolumn{4}{|l|}{ ATB group, mm } \\
\hline$<5$ & $31(65.96)$ & $16(34.04)$ & 34.04 \\
\hline $5-9$ & $14(56.00)$ & $11(44.00)$ & 56.00 \\
\hline $10-19$ & $64(81.01)$ & $15(18.99)$ & 81.01 \\
\hline$\geq 20$ & $27(77.14)$ & $8(22.86)$ & 77.14 \\
\hline \multicolumn{4}{|l|}{ Non-TB group, mm } \\
\hline$<5$ & $6(9.38)$ & $58(90.62)$ & 90.62 \\
\hline $5-9$ & $4(33.33)$ & $8(66.67)$ & 33.33 \\
\hline $10-19$ & $16(44.44)$ & $20(55.56)$ & 44.44 \\
\hline$\geq 20$ & $4(30.77)$ & $9(69.23)$ & 30.77 \\
\hline
\end{tabular}

TST, tuberculin skin test; TB, tuberculosis; ATB, active tuberculosis.

Table VII. Comparison of performances between TST and T-SPOT.TB tests and their combinations.

\begin{tabular}{lcccrrr}
\hline Tests & Sensitivity, $\%$ & Specificity, $\%$ & PPV, $\%$ & NPV, $\%$ & LR+ & LR- \\
\hline T-SPOT.TB & 76.66 & 76.37 & 86.08 & 63.18 & 3.24 & 0.31 \\
TST & 75.56 & 51.15 & 69.50 & 57.66 & 1.53 & 0.52 \\
T-SPOT.TB and TST (parallel) & 86.46 & 56.04 & 71.37 & 80.56 & 1.72 & 0.17 \\
T-SPOT.TB and TST (serial) & 67.72 & 79.67 & 82.73 & 57.06 & 3.31 & 0.52 \\
\hline
\end{tabular}

TST, tuberculin skin test; PPV, positive predictive value; NPV, negative predictive value; LR+, positive likelihood ratio; LR-, negative likelihood ratio.

positive rates of the T-SPOT.TB assay in the four groups were shown to exhibit an increasing trend $\left(\chi^{2}=38.0113, \mathrm{P}<0.001\right.$; Table VI).

Efficiency of combining the T-SPOT.TB assay and TST results. Further estimations were undertaken to assess whether the diagnostic efficiency was improved by combining the results from the T-SPOT.TB assay and TST. The results were combined in a parallel manner, where a positive result was assumed when either test was positive, and a negative result was assumed when both tests were negative. In addition, the results were combined in a serial manner, in which a positive result was assumed when both tests were positive and a negative result was inferred when either test was negative. Parallel testing increased the sensitivity of the T-SPOT.TB assay to $86.46 \%$ when combined with the TST; however, this was on the expense of a considerable increase in the false-positive rate (the specificity declined to $56.04 \%$ ). By contrast, serial testing increased the specificity of the T-SPOT.TB assay to $79.67 \%$ when combined with TST; however, the sensitivity decreased to $67.72 \%$ (Table VII). With sole application of the T-SPOT.TB test, the specificity was $76.37 \%$ (95\% CI, 70.20-82.54\%), the PPV was $86.08 \%$ (95\% CI, 89.93-82.23\%), the NPV was $63.18 \%(95 \% \mathrm{CI}$, 66.43-63.15\%), the LR+ was 3.24 and the LR- was 0.31 for an ATB diagnosis.

\section{Discussion}

The diagnosis of TB in patients with negative bacteriological results remains a problem that requires resolving in clinical practice. Nonetheless, since the cross-reaction of the TST with the $B C G$ vaccination attenuates the specificity, alternative, fast and specific diagnostic tools are required to replace the TST. The aim of the present study, performed in a country with a high prevalence of $\mathrm{TB}$ and $\mathrm{BCG}$ vaccination rates, was to determine the efficiency of the T-SPOT.TB test for diagnosing TB in routine clinical practice.

In the present study, the sensitivity values of the T-SPOT.TB test for the diagnosis of active PTB and EPTB were determined 
to be 77.97 and $69.23 \%$, respectively, which were markedly lower compared with previously reported studies (12-14). The lower sensitivity obtained in the present analysis may be due to the patients included in the sample population, since they were undergoing anti-TB treatment. The positive rate of the T-SPOT.TB assay in the ATB patients undergoing anti-TB treatment was significantly lower when compared with the patients not receiving treatment $(\mathrm{P}=0.002)$. In addition, an increased treatment time was found to be associated with a lower positive rate. This phenomenon may be associated with a decreased antigen burden, which is in agreement with several previous studies (15-18). In order to confirm the hypothesis that the T-SPOT.TB assay results may be directly associated with the actual bacterial load, the correlation between sensitivity and disease severity, plus the smear grade, was further investigated. The sensitivity gradually decreased with a reduction in disease severity and a decreasing smear grade; however, no statistically significant differences were observed, which may be due to the small sample size included in the present study. Ribeiro et al demonstrated that only $10 \%$ of individuals with a baseline reactive test reverted to negative results following completion of 24 weeks of treatment (17). Thus, the hypothesis that the T-SPOT.TB assay may serve as an effective predictor of therapeutic efficacy in ATB patients undergoing anti-TB treatment requires further investigation.

There has been considerable debate with regard to the diagnostic value of IGRAs for EPTB. Fan et al recorded a pooled sensitivity of $90 \%$ (95\% CI, 86-93\%) (13). However, Cho et al reported that the T-SPOT.TB assay was more sensitive in patients with chronic forms of EPTB, such as lymph node or osteoarticular TB $(93 \%)$, as compared with patients with acute EPTB forms, such as TB meningitis or miliary TB (79\%) (19). Furthermore, Liao et al reported an overall sensitivity of $79.8 \%$, with the sensitivity ranging between $100 \%$ for tuberculous meningitis, tuberculous pericarditis and intestinal $\mathrm{TB}, 95 \%$ for lymphadenitis and $42.9 \%$ for tuberculous peritonitis (20). The results of the present study revealed an overall sensitivity of $69.23 \%$, which was considerably lower than the value for PTB alone, and the sensitivity values ranged between $78.79 \%$ for tuberculous pleurisy to $69.23 \%$ for tuberculous lymphadenitis and $16.67 \%$ for osteoarticular TB. The difference in sensitivity between the affected sites was determined to be statistically significant $(\mathrm{P}=0.01)$. Therefore, the observations of the present study add strength to the hypothesis that IFN- $\gamma$-producing T-cell responses vary according to the location of the disease; however, future investigation is required to elucidate this hypothesis further. The positivity rate of $23.63 \%$ for the T-SPOT.TB assay in the non-TB group is consistent with the estimation that one third of the world's population is infected with M.tuberculosis, according to the estimations of the World Health Organization (21).

In conclusion, the results of the present study indicate that the T-SPOT.TB assay may be a promising test for the diagnosis of active PTB due to the improvement in specificity observed in a BCG-vaccinated population. Thus, the test may replace the TST in clinical practice. As the performance of the test was shown to be associated with anti-TB treatments, for patients undergoing anti-TB treatment, the T-SPOT.TB assay may be of diagnostic value for the assessment of treatment outcomes.

\section{Acknowledgements}

This study was supported by a grant from the National Key Scientific and Technical Program (no. 2013ZX10003009).

\section{References}

1. Moyle PM and Toth I: Modern subunit vaccines: Development, components and research opportunities. ChemMedChem 8: 360-376, 2013

2. Brodie D and Schluger NW: The diagnosis of tuberculosis. Clin Chest Med 26: 247-271, 2005.

3. Richeldi L: An update on the diagnosis of tuberculosis infection. Am J Respir Crit Care Med 174: 736-742, 2006.

4. Jiang W, Shao L, Zhang Y, et al: High-sensitive and rapid detection of Mycobacterium tuberculosis infection by IFN-gamma release assay among HIV-infected individuals in BCG-vaccinated area. BMC Immunol 10: 31, 2009.

5. Diel R, Loddenkemper R and Nienhaus A: Evidence-based comparison of commercial interferon-gamma release assays for detecting active TB: A metaanalysis. Chest 137: 952-968, 2010.

6. Lalvani A and Pareek M: Interferon gamma release assays: Principles and practice. Enferm Infecc Microbiol Clin 28: 245-252, 2010.

7. Ewer K, Deeks J, Alvarez L, et al: Comparison of T-cell-based assay with tuberculin skin test for diagnosis of Mycobacterium tuberculosis infection in a school tuberculosis outbreak. Lancet 361: 1168-1173, 2003.

8. Andersen P, Munk ME, Pollock JM and Doherty TM: Specific immune-based diagnosis of tuberculosis. Lancet 356: 1099-1104, 2000.

9. Yang CH, Chan PC, Liao ST, et al: Strategy to better select HIV-infected individuals for latent TB treatment in BCG-vaccinated population. PLoS One 8: e73069, 2013.

10. Yu Y, Zhao X, Wang W, et al: Diagnostic performance of interferon-gamma releasing assay in HIV-infected patients in China. PLoS One 8: e70957, 2013.

11. Singanayagam A, Manalan K, Sridhar S, et al: Evaluation of screening methods for identification of patients with chronic rheumatological disease requiring tuberculosis chemoprophylaxis prior to commencement of TNF- $\alpha$ antagonist therapy. Thorax 68: 955-961, 2013

12. Dai Y, Feng Y, Xu R, Xu W, Lu W and Wang J: Evaluation of interferon-gamma release assays for the diagnosis of tuberculosis: An updated meta-analysis. Eur J Clin Microbiol Infect Dis 31: 3127-3137, 2012.

13. Fan L, Chen Z, Hao XH, Hu ZY and Xiao HP: Interferon-gamma release assays for the diagnosis of extrapulmonary tuberculosis: A systematic review and meta-analysis. FEMS Immunol Med Microbiol 65: 456-466, 2012.

14. Feng Y, Diao N, Shao L, et al: Interferon-gamma release assay performance in pulmonary and extrapulmonary tuberculosis. PLoS One 7: e32652, 2012.

15. Domínguez J, De Souza-Galvão M, Ruiz-Manzano J, et al: T-cell responses to the Mycobacterium tuberculosis-specific antigens in active tuberculosis patients at the beginning, during and after antituberculosis treatment. Diagn Microbiol Infect Dis 63: 43-51, 2009.

16. Bosshard V, Roux-Lombard P, Perneger T, et al: Do results of the T-SPOT.TB interferon-gamma release assay change after treatment of tuberculosis? Respir Med 103: 30-34, 2009.

17. Ribeiro S, Dooley K, Hackman J, et al: T-SPOT.TB responses during treatment of pulmonary tuberculosis. BMC Infect Dis 9: 23, 2009.

18. Chee CB,KhinMar KW, Gan SH, et al: Tuberculosis treatment effect on T-cell interferon-gamma responses to Mycobacterium tuberculosis-specific antigens. Eur Respir J 36: 355-361, 2010.

19. Cho OH, Park KH, Kim SM, et al: Diagnostic performance of T-SPOT.TB for extrapulmonary tuberculosis according to the site of infection. J Infect 63: 362-369, 2011.

20. Liao CH, Chou CH, Lai CC, et al: Diagnostic performance of an enzyme-linked immunospot assay for interferon-gamma in extrapulmonary tuberculosis varies between different sites of disease. J Infect 59: 402-408, 2009.

21. World Health Organization (WHO): The global plan to stop TB 2011-2015. Geneva, WHO, 2010. 